\title{
Domestication de nouvelles espèces d'intérêt piscicole en Amazonie
}

\author{
Jesus Nuñez ${ }^{1,2}$ \\ 1 IRD \\ GAMET \\ BP 5095 \\ 361, rue J.-F.-Breton \\ 34196 Montpellier cedex 05 \\ France \\ <nunez@ird.fr> \\ 2 UNFV-FOPCA \\ 350, Calle Roma \\ Miraflores \\ Lima \\ Pérou
}

\begin{abstract}
Résumé
La biodiversité ichthyologique en Amazonie est parmi les plus importantes de la planète avec environ 2500 espèces recensées et une estimation de plus de 5000 au total, voire 6000 si l'on considère l'Amérique du Sud, l'Amérique centrale et les Caraỉbes. Malgré cette grande diversité ichthyologique, la production piscicole des espèces "introduites " est encore supérieure à celle des espèces autochtones. L'idée de développer des espèces locales s'est finalement imposée devant le constat d'une pêche d'eau douce en stagnation et parfois même en régression pour certaines des espèces les plus demandées sur les marchés. On observe depuis plus d'une dizaine d'années une hausse continue de la production piscicole d'espèces amazoniennes, Colossoma macropomum, notamment Piaractus brachypomus et leurs hybrides particulièrement en vogue au Brésil et en Colombie. À l'heure actuelle, quelques dizaines d'espèces ont été identifiées comme présentant des caractéristiques intéressantes pour la pisciculture et, parmi elles, environ une dizaine fait réellement l'objet d'un processus de domestication même si l'état d'avancement est encore très variable suivant les espèces. Parallèlement, au développement de la production pour la consommation humaine, on constate l'émergence de l'élevage d'espèces ornementales et des actions de repeuplement d'espèces indigènes. Compte tenu des pratiques comme les introductions d'espèces et la production d'hybrides non stériles, le développement de la pisciculture devra s'accompagner de règles de conduite claires tant du point de vue des ressources hydriques que biologiques.
\end{abstract}

Mots clés : Amazonie ; domestication ; espèce nouvelle ; pisciculture ; poisson.

Thèmes : pêche et aquaculture ; production animale ; ressource naturelle et environnement.

\section{Abstract \\ Domestication of new economically important Amazonian fish species}

The diversity of fish fauna in Amazonia is one of the most extensive of the planet with approximately 2,500 species listed and an estimate of more than 5,000 and even 6,000 if one considers South America, Central America and the Caribbean. In spite of this great ichthyological diversity, the pisciculture production of "introduced" or "alien" species is higher than that of autochthonous ones. The necessity of developing fish farming of local species came to light upon consideration of the stagnation of fresh water fish captures and even the regression for some of the most highly required species on the Amazonian and South American markets. For over ten years one has observed a continuous rise in the pisciculture production of Amazonian species, particularly Colossoma macropomum, Piaractus brachypomus and their hybrids, and more recently an ornamentalfish-based aquaculture. At present, a few tens of species for consumption have been identified as good candidates for fish farming and among them approximately ten are being subjected to a process of domestication even if the progress reports remain highly contrasted according to the species. The necessary and desirable development of fish farming will have to be accompanied by clear and applicable codes of conduct in order to allow sustainable development of this activity, respectful of the environment and especially hydrous and biological resources.

Key words: Amazonia; aquaculture; domestication; fish culture; new species.

Subjects: animal production; fishing and aquaculture; natural resources and environment.

Tirés à part : J. Nuñez 
Amazonie, avec plus de $6000000 \mathrm{~km}^{2}$, représente un territoire d'environ huit fois la France et concerne huit pays sud-américains, le Brésil à lui seul possédant plus de la moitié de la superficie totale (figure 1). La diversité ichthyologique y est parmi les plus importantes de la planète, avec environ 2500 espèces recensées en Amazonie (Junk et al., 2007) et une estimation de plus de 5000 espèces au total, voire 6000 (Lundberg et al., 2000) si l'on intègre l'Amérique du Sud, l'Amérique centrale et les Caraibes. Parmi cette grande diversité, plusieurs dizaines d'espèces ont été, à des degrés divers, "testées " pour leur aptitude à l'élevage au cours des 50 dernières années. Cependant, la valorisation de nouvelles espèces passe par un long processus de domestication et de sélection. Le manque de connaissances sur les espèces amazoniennes ou plus généralement sud-américaines a été, en grande partie, à l'origine de l'introduction sur le continent sud-américain d'espèces allochtones dont les cycles d'élevage étaient déjà maîtrisés : la carpe (Cyprinus carpio), la truite (Onchorbynchus mykiss), les saumons (Salmo salar et Onchorhynchus kisutch), le silure africain (Clarias gariepinus) et le tilapia (Oreochromis niloticus), entre autres. Cette pratique a permis une production immédiate sans avoir à entreprendre des recherches sur les espèces autochtones encore mal connues tant du point de vue de leur biologie que de leur potentiel aquacole. Il faut noter que même actuellement au Brésil, le tonnage cumulé de la production piscicole des espèces "introduites " est supérieur à celui des espèces autochtones (les carpes, Cyprinus carpio et Hypophthalmichthys sp., et tilapias, Oreochromis sp., représentaient plus de la moitié de la production piscicole en 2003, source FAO). L'idée de développer des espèces locales s'est finalement imposée devant le constat d'une pêche d'eau douce en stagnation et parfois même en régression pour certaines espèces les plus demandées (Petrere et al., 2004; FAO, 2005) et aussi, en raison du marché important que représentent les espèces traditionnelles bien connues et appréciées par les consommateurs amazoniens et sud-américains.

Le développement de la pisciculture en Amérique latine et aux Caraibes connaît une croissance constante du même ordre (8 à $10 \%$ ) que la croissance mondiale, mais en volume de production, elle ne représente encore qu'un peu plus de

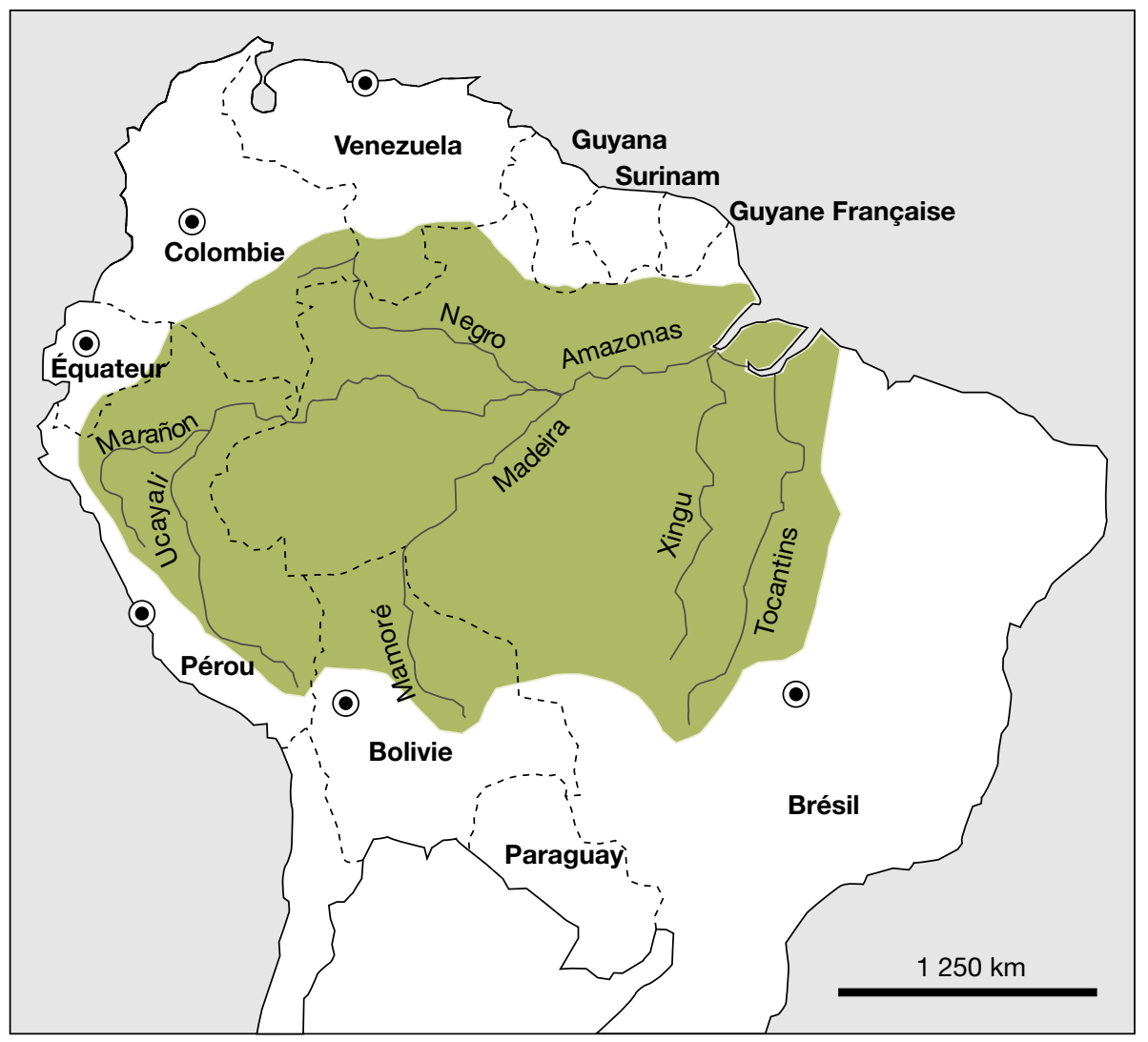

Figure 1. Limites géographiques du Bassin amazonien.

Figure 1. Geographical extension of the Amazon basin.

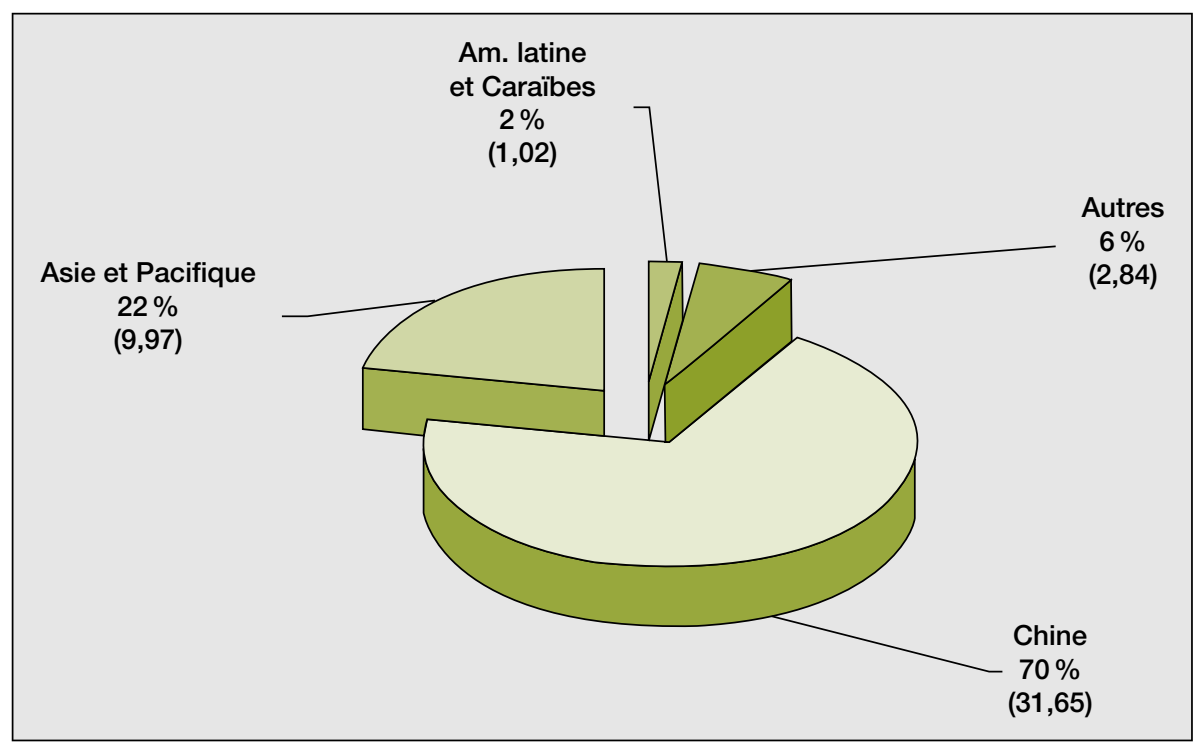

Figure 2. Répartition de la production piscicole mondiale (millions de tonnes) en 2004.

Figure 2. World pisciculture production (million tonnes) by main areas in 2004. 


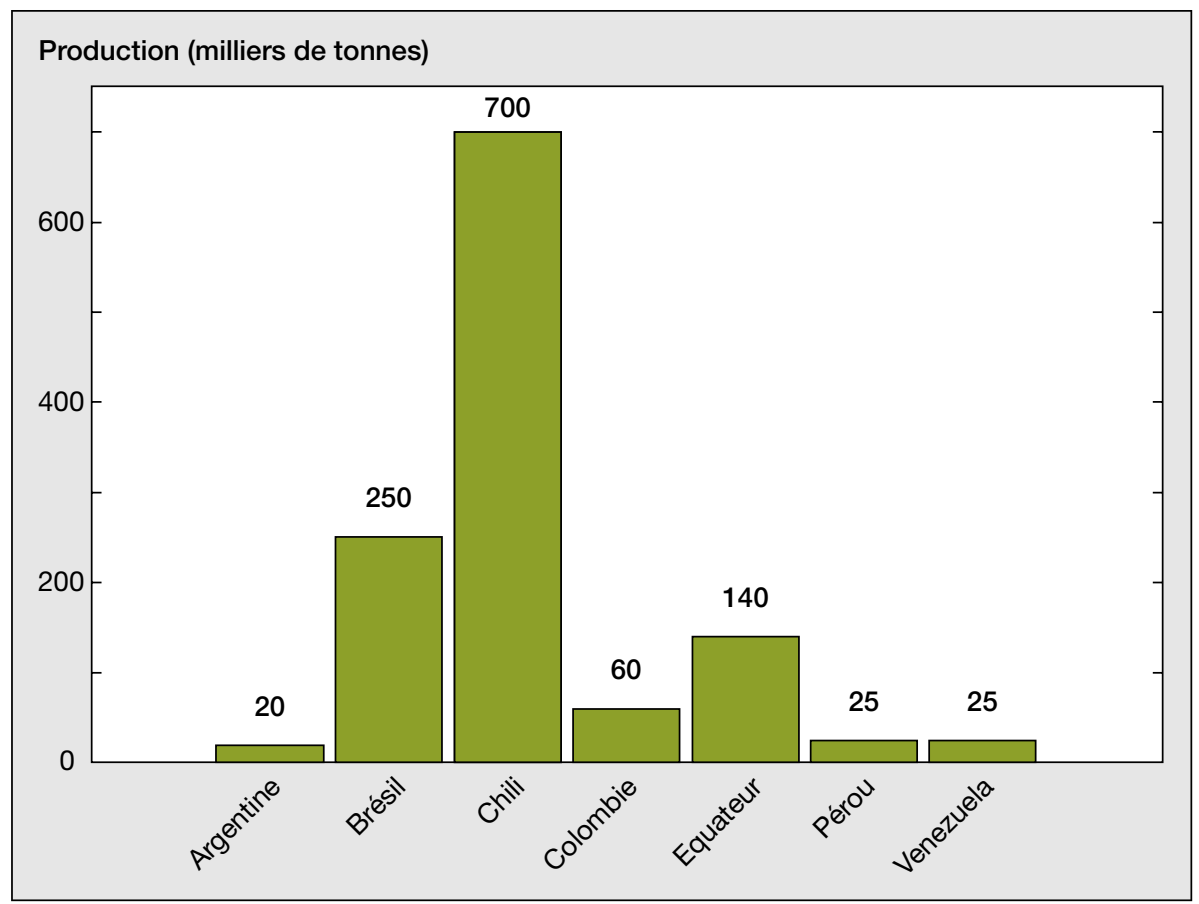

Figure 3. Production piscicole totale (poissons marins et continentaux) pour les principaux pays d'Amérique du Sud en 2006 (source FAO).

Figure 3. Pisciculture (marine and inland fish production) of the main south American countries in 2006.

$2 \%$ de la production mondiale (figure 2 ), alors que le potentiel est considérable. La production piscicole des principaux pays sud américains est encore très contrastée (figure 3), le Chili étant le principal acteur avec l'élevage du saumon destiné presque exclusivement à l'exportation. Le développement de la pisciculture est significatif depuis plus d'une quinzaine d'années, notamment au Brésil où le niveau de production atteint désormais environ $35 \%$ des débarquements de la pêche continentale (figure 4), mais la part de la pisciculture croît également en Colombie au Venezuela et au Pérou.

À côté des espèces introduites et mondialement connues (carpes, Cyprinus carpio, Ctenopharbyngodon idella et Hypophthalmichthys sp., et tilapias, Oreochromis sp.), on observe, depuis une dizaine d'années, une hausse continue de la production piscicole d'espèces parmi les premières testées, Colossoma macropomum, notamment Piaractus brachypomus et leurs hybrides. Les espèces testées (espèces amazoniennes mais aussi des bassins du Paraná-Paraguay et du Sao Francisco) au cours des dernières décennies ont été choisies généralement en raison de leur taille maximale, qui présupposait une bonne croissance en élevage (Arapaima, Salminus, Hoplias, Conorynchus, Bra- chyplatystoma, Pseudoplatystoma, etc.) et/ou de leur acceptation sur le marché local (Tucunarés, Cichla sp.) ou ayant un régime alimentaire omnivore ou détritivore (Colossoma, Piaractus, Leporinus, Brycon, Prochilodus), favorisant la couverture des besoins alimentaires à l'aide d'intrants moins coûteux et plus facilement disponibles. À l'heure actuelle, on peut compter sur quelques dizaines d'espèces identifiées parmi les genres cités comme présentant des caractéristiques intéressantes pour l'élevage de poissons de consommation. Parmi les caractéristiques qui ont permis de cibler ces espèces, on peut citer principalement : le marché local potentiel, la facilité de reproduction et d'obtention d'alevins, la croissance et la résistance au stress et aux manipulations. Au sein de cette présélection (tableau 1), environ une dizaine font réellement l'objet d'un processus de domestication (tableau 2), même si l'état d'avancement est encore très variable suivant les espèces. Dans ce groupe, on peut considérer que deux espèces présentent un potentiel avéré en termes de production (tableau 2) : C. macropomum, P. brachypomus et leurs hybrides. Le développement de leur élevage a été rendu possible, particulièrement, en raison de leur rusticité. En effet, elles peuvent être alimen- tées à partir de différentes sources végétales (fruits, graines) ou d'aliments composés, généralement à faible taux de farine de poisson, dans des contextes de production extensif, semi-intensif en étangs ou intensif en cages. La progression de leur production au niveau de la région passe maintenant par une phase de domestication-sélection pour améliorer les paramètres biotechniques. Quant aux autres espèces, elles nécessitent encore des avancées significatives dans les domaines de la reproduction, l'élevage larvaire et l'alimentation pour parvenir à contribuer durablement au développement de la production piscicole Amazonienne. Il faut signaler, également, l'émergence d'une production piscicole d'espèces ornementales destinées principalement, pour le moment, au marché interne, comme les scalaires, les tétras, des petits Siluriformes (Pimelodella, Hoplosternum, Dianemas, Ancistrus, Hypostomus) et quelques Anostomidae (Anostomus, Leporinus). Les individus sauvages sont généralement exportés en raison d'une plus grande valeur marchande. Cependant, à court terme, cette pratique met en péril la survie de la plupart des espèces dans le milieu naturel pour les trois principaux exportateurs du continent sud-américain (Brésil, Colombie, Pérou).

Plusieurs des espèces cultivées font également l'objet de programmes de repeuplement (Prochilodus, Leporinus, Piaractus, Salminus, Brycon, Cichla) notamment dans le bassin du Sao Francisco, davantage touché par la surpêche que les autres bassins, la pollution et l'établissement de barrages hydroélectriques, réduisant considérablement la reproduction des espèces migratrices.

Ces programmes, bien que fortement médiatisés, sont souvent critiqués en raison de la faible qualité des alevins relâchés (faible diversité génétique) et leur mauvaise adaptation aux conditions de survie dans des milieux parfois pollués et/ou fortement anthropisés.

\section{Présentation des espèces}

\section{Arapaima gigas}

Arapaima gigas est une des espèces emblématiques du bassin amazonien, en raison notamment de la taille qu'elle peut 


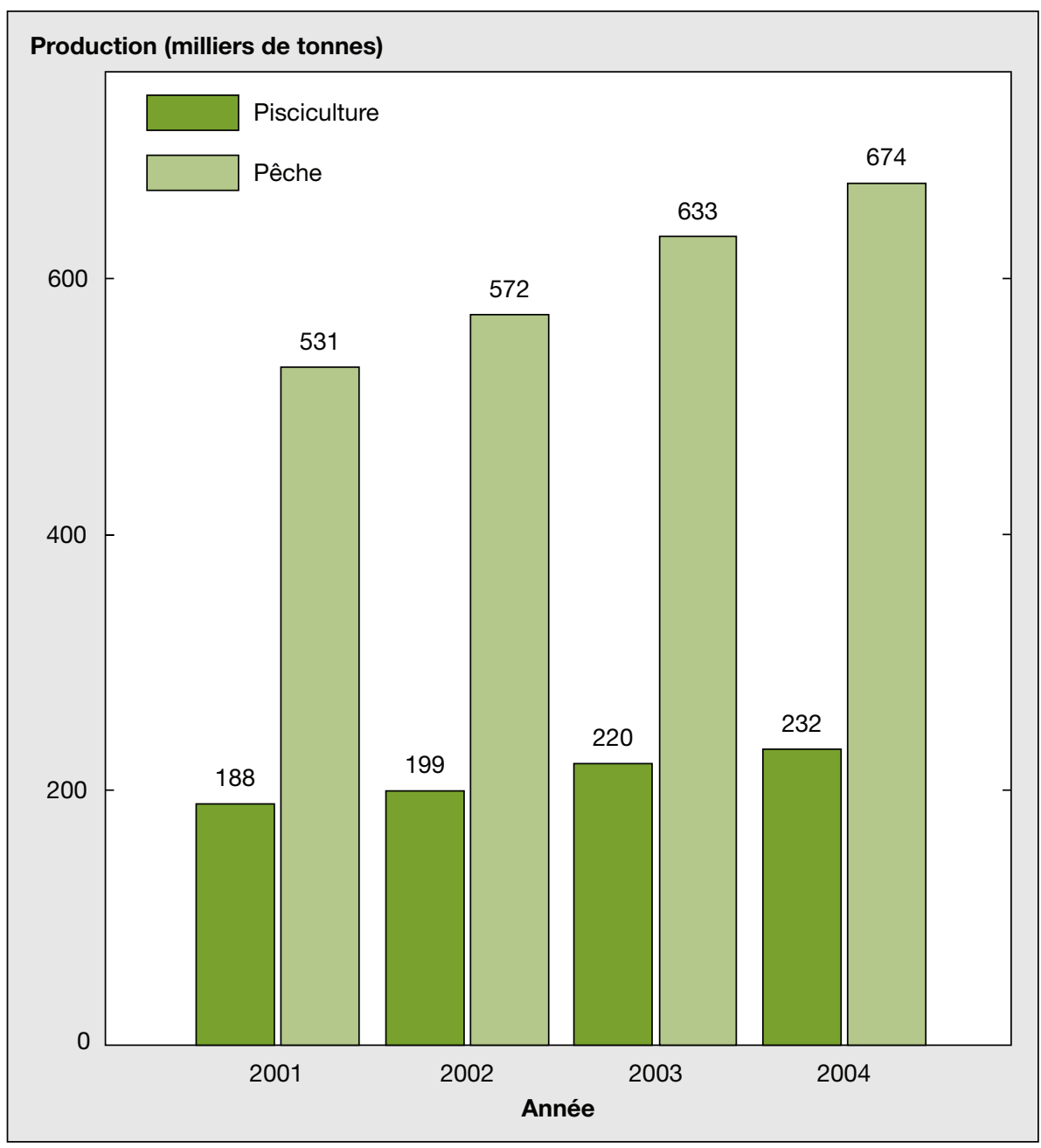

Figure 4. Parts des productions piscicoles et de la pêche continentale et marine en 2004 au Brésil (source FAO).

Figure 4. Fish culture and fisheries production (freshwater and marine species) in Brazil for 2004 (FAO source).

atteindre; on a rapporté des captures encore récentes de poissons de plus de trois mètres et $200 \mathrm{~kg}$, et, dans les années 1900, on a observé des tailles de capture de plus de 4,50 m (Henn, 1912). Ce n'est donc pas étonnant qu'il soit apprécié par les populations locales en raison de la quantité de nourriture qu'il représente en une seule prise. Il était déjà parmi les espèces les plus appréciées des habitants de la région au $\mathrm{xIX}^{\mathrm{e}}$ siècle, connu comme la "morue de l'Amazone "(Tyler, 1894) en raison de sa méthode de conservation en rouleaux salés et séchés au soleil. Aujourd'hui, après une quinzaine d'années de pêche intensive, l'espèce est en danger dans son habitat naturel. Des réserves ont été mises en place au Brésil ainsi qu'au Pérou, et il existe une volonté de gérer de façon durable cette ressource, notamment en réintroduisant l'espèce dans des zones favorables et contrôlables à partir d'alevins produits en pisciculture. De nombreux essais de "domestication" ont été entrepris au cours des 50 dernières années, malheureusement, la biologie encore mal connue de l'espèce et des caractéristiques très particulières de reproduction (formation de nids, surveillance parentale) n'ont pas permis obtenir des succès significatifs dans la production massive d'alevins. Parmi les points de blocage non encore résolus, le plus important est certainement la maîtrise de la reproduction en captivité. Différents essais ont été et sont encore menés au Brésil, Colombie et Pérou (Pereira-Filho et al., 2003 ; Rebaza Alfaro et al., 2003 ; Saavedra Rojas et al., 2005) pour tenter de définir les meilleures conditions pour une reproduction " naturelle " de A. gigas. Les meilleurs rendements de reproduction obtenus jusqu'à aujourd'hui en étangs de plusieurs milliers de mètres carrés sont en moyenne de 2000 et vont exceptionnellement jusqu'à 5000 jeunes alevins par ponte. Néanmoins, même si plusieurs couples sont présents, en général un seul couple se reproduit une seule fois dans l'année. La constitution des lots de géniteurs se fait au hasard, car il n'y a pas de caractère morphologique extérieur permettant de caractériser les sexes. Récemment, notre équipe a mis au point un sexage des géniteurs basé sur la vitellogénine plasmatique qui devrait permettre d'améliorer la constitution des stocks de reproducteurs (ChuKoo et al., 2008). Jusqu'à présent, il fallait un minimum de quatre à six individus par structure pour pouvoir espérer la formation d'au moins un couple susceptible de se reproduire. Les alevins sont récoltés généralement après 15 jours à trois semaines à une taille d'environ $5 \mathrm{~cm}$ et à un poids de $1 \mathrm{~g}$. Leur prix est d'environ $2 \$$ pour un alevin de $5 \mathrm{~cm}$ et peut atteindre $10 \$$ ou plus en fonction de la taille. L'élevage se fait ensuite, généralement, en étang, en polyculture et plus récemment en cages et en enclos avec une alimentation complètement artificielle sous forme d'aliment extrudé contenant $40 \%$ de protéines. Le rendement économique de l'élevage intensif n'est pas encore complètement caractérisé, mais plusieurs études ont montré le fort potentiel de croissance permettant d'obtenir des poissons de plus de $10 \mathrm{~kg}$ en 12 mois d'élevage, ce qui constitue un avantage important sur beaucoup d'autres espèces. L'évaluation des volumes produits est très difficile, car leur vente se fait en petites quantités tout au long de l'année.

Le développement de l'élevage du pirarucu ou paiche passe par une meilleure gestion de la reproduction " naturelle " et en particulier par le sexage des géniteurs, afin de constituer des couples et d'augmenter ainsi les taux de reproduction dans des structures plus nombreuses et plus petites en surface. Par ailleurs, des essais de stimulation de la reproduction " naturelle " ont été entrepris, notamment en utilisant des traitements à base d'extraits hypophysaires de carpe et d'injections d'implants de LH-RHa. Pour le moment, aucun de ces traitements n'a donné de résultat significatif, mais cette approche mérite d'être poursuivie en l'associant à la détermination de critères physiologiques et comportementaux. 
Tableau 1. Principales espèces amazoniennes ayant fait l'objet d'une utilisation en pisciculture et principaux critères de sélection.

Table 1. Main cultivated species in Amazonia and principal species selection criteria.

\begin{tabular}{|c|c|c|c|c|c|c|c|}
\hline Nom & $\underset{(\mathrm{cm})^{*}}{\mathrm{~L}_{\max }}$ & $\begin{array}{l}P_{\max } \\
(\mathbf{k g})^{*}\end{array}$ & $\begin{array}{c}\text { Acceptation } \\
\text { par la } \\
\text { population }\end{array}$ & $\begin{array}{l}\text { Reproduction } \\
\text { induite }\end{array}$ & $\begin{array}{c}\text { Résistance } \\
\text { à la } \\
\text { manipulation }\end{array}$ & $\begin{array}{l}\text { Facilité de } \\
\text { production } \\
\text { d'alevins }\end{array}$ & $\begin{array}{l}\text { Régime } \\
\text { alimentaire }\end{array}$ \\
\hline Arapaima gigas & 400 & 250 & +++ & - & ++ & - & Pisciv. \\
\hline Brachyplatystoma filamentosum & 360 & 200 & ++ & \pm & \pm & \pm & Pisciv. \\
\hline Brachyplatystoma rousseauxii & 180 & 100 & ++ & \pm & \pm & \pm & Pisciv. \\
\hline Phractocephalus hemioliopterus & 134 & 44 & + & \pm & + & - & Pisciv. \\
\hline Pseudoplatystoma tigrinum & 130 & 18 & +++ & + & ++ & + & Pisciv. \\
\hline Pseudoplatystoma fasciatum & 120 & 15 & +++ & + & ++ & + & Pisciv. \\
\hline Colossoma macropomum & 108 & 40 & +++ & ++ & ++ & +++ & Omniv./Phyt. \\
\hline Salminus maxillosus & 100 & 31 & + & \pm & \pm & + & Pisciv. \\
\hline Hoplias sp. & 100 & 40 & + & \pm & + & + & Pisciv. \\
\hline Cichla sp. & 90 & 12,5 & ++ & - & \pm & - & Pisciv. \\
\hline Piaractus brachypomus & 88 & 25 & +++ & ++ & ++ & +++ & Omniv./Phyt. \\
\hline Plagioscion squamosissimus & 80 & 4,5 & ++ & \pm & - & \pm & Pisciv. \\
\hline Brycon sp. & 58 & 4 & ++ & + & \pm & ++ & Omniv./Phyt. \\
\hline Prochilodus sp. & 50 & 3 & ++ & + & \pm & ++ & Détritivore \\
\hline Leporinus sp. & 40 & 1,5 & ++ & \pm & \pm & + & Omniv./Phyt. \\
\hline Rhamdia quelen & 35 & 4 & + & + & + & ++ & Omniv./Pisc. \\
\hline
\end{tabular}

$L_{\max }$ : Longueur maximale; $P_{\max }$ : Poids maximum ; Pisciv. : Piscivore ; Omniv. : Omnivore ; Phyt. : Phytophage.

* Sources : IBAMA (Brasil), INCODER (Colombia), UNET (Venezuela), IIAP (Perú), FishBase (www.fishBase.org).

\section{Brycon sp.}

Cette espèce peut être considérée comme importante, au moins à l'échelle de l'Amérique du Sud, car elle constituait une ressource abondante pour la pêche et donc pour la consommation des populations locales. Actuellement, les stocks naturels ont fortement diminué, sans doute davan- tage en raison de la modification de l'écosystème que de la pression de pêche ellemême. La construction de barrages notamment a fortement freiné les migrations reproductives vers l'amont des cours d'eau au début de la période de crue (Welcomme, 1985 ; Mateus et Estupiñán, 2002). Toutes ces espèces ont un régime omnivore et présentent donc de bonnes caractéristiques pour l'élevage en pisciculture malgré la propension au cannibalisme à l'état larvaire (Baras et al., 2000). Leur élevage connaît un réel développement (tableau 2), surtout au Brésil, avec des résultats encourageants associés à un travail de recherche important sur les bases biologiques de la reproduction et de l'élevage larvaire (Mazzotti Santamaria

\section{Tableau 2. Principales espèces en cours de domestication en Amazonie.}

Table 2. Main species in " domestication " process in Amazonia.

\begin{tabular}{|c|c|c|c|c|c|}
\hline Ordre & Famille & Genre & Espèce & Noms locaux & Production $(2005)^{a}$ \\
\hline Ostéoglossiformes & Arapaimidae & Arapaima & gigas & Pirarucu, Paiche & $<500$ \\
\hline Characiformes & Characidae & Brycon & amazonicus & Sabalo & Brycon sp. 7000 \\
\hline Characiformes & Characidae & Brycon & orbignyanus & Piracanjuba, Sabalo & \\
\hline Characiformes & Characidae & Brycon & moorei & Piracanjuba, Sabalo & \\
\hline Characiformes & Characidae & Colossoma & macropomum & Pacú, Tambaquí, Gamitana & $\begin{array}{l}45000 \\
\text { Hybrides } 15000\end{array}$ \\
\hline Characiformes & Characidae & Piaractus & brachypomus & Tambaquí, Pacú, Paco & 14000 \\
\hline Siluriformes & Pimelodidae & Pseudoplatystoma & fasciatum & Surubí, Sorubim, Doncella & Pseuplatystoma sp. \\
\hline Siluriformes & Pimelodidae & Pseudoplatystoma & tigrinum & $\begin{array}{l}\text { Cacharra, Chuncuina, } \\
\text { Zungaro }\end{array}$ & 1800 \\
\hline Siluriformes & Hepteridae & Rhamdia & quelen & $\begin{array}{l}\text { Jundiá, bagre negro, bagre } \\
\text { sapo }\end{array}$ & 800 \\
\hline
\end{tabular}

Les productions sont indiquées en tonnes.

a Sources : IBAMA (Brasil), INCODER (Colombia), UNET (Venezuela), IIAP (Perú). 
et Araujo Antunes, 1999; Ganeco et al., 2001 ; de Borba et al., 2003 ; Feiden et Hayashi, 2005; Freato et al., 2005). Les techniques développées permettent l'obtention de la reproduction par induction hormonale et fécondation artificielle. Généralement, pour l'induction de la maturation ovocytaire et l'ovulation, on utilise encore davantage les extraits hypophysaires de carpe que les analogues du LH-RH en deux ou trois injections successives $(0,25 \mathrm{mg} / \mathrm{kg}$ et après 24 heures $0,5 \mathrm{mg} / \mathrm{kg}$, puis $5 \mathrm{mg} / \mathrm{kg} 8$ à 12 heures plus tard). Les mâles reçoivent en général de 20 à $50 \%$ de la dose administrée aux femelles en une seule injection en même temps que la première injection des femelles. La survie larvaire constitue le principal point de blocage avec le problème de cannibalisme observé dès environ 20 heures postéclosion (Baras et al., 2000).

\section{Colossoma macropomum, Piaractus brachypomus}

Ces deux espèces sont historiquement les premières espèces natives à avoir été utilisées en pisciculture après avoir obtenu l'induction de la ponte en captivité, en utilisant la technique d'hypophysation (Woynarovich et Horváth, 1981) pour les espèces tropicales. Le niveau de production actuel en Amérique latine est de plus de 75000 t, et il représente la plus grande production d'espèces natives amazoniennes. Ces Characidae sont rustiques et supportent donc bien la captivité et les manipulations. Leur régime alimentaire omnivore, avec une forte consommation de fruits et de baies dans le milieu naturel, leur permet de s'adapter en élevage à une alimentation à base de granulés ou d'aliment extrudé à faible teneur en farine de poisson (5 à $15 \%$ ). La production d'alevins issus de géniteurs sauvages ou nés en captivité est bien maîtrisée. Les meilleures croissances en étang (densité de 1 à 5 poissons par $10 \mathrm{~m}^{2}$ ) se situent autour de $1 \mathrm{~kg}$ en six à dix mois pour $C$ macropomum et 500 à $600 \mathrm{~g}$ pour $P$. brachypomus avec un aliment composé contenant environ $28 \%$ de protéines (dont environ $80 \%$ d'origine végétale) distribué à raison de $3 \%$ de la biomasse corporelle. En cagesenclos, dans les meilleures conditions de température (autour de $28^{\circ} \mathrm{C}$ ), le poids de $1 \mathrm{~kg}$ est atteint après environ 10 à 12 mois d'élevage à des densités de 25 à 50 poissons par mètre cube pour C. macropomum (Araujo-Lima et de Carvalho Gomes, 2005).
Il faut signaler également l'utilisation, principalement au Brésil et en Colombie, de leur hybride (Tambacú) entre femelles de $C$. macropomum et mâles de P. mesopotamicus (originaires des bassins du Sao Francisco et du Paraná) qui, en raison d'une meilleure résistance aux variations annuelles de température, est utilisé de façon importante dans le Pantanal et le Nordeste. L'utilisation de cet hybride en Amazonie reste encore limitée, mais elle pose le problème de la pollution génétique de l'espèce sauvage parentale ( $C$. macropomum) dans la mesure où différents travaux ont rapporté que les hybrides obtenus ne sont pas stériles (Calcagnotto et al., 1999). Par conséquent, l'impact de leur échappement dans le milieu naturel reste à déterminer.

\section{Pseudoplatystoma fasciatum, Pseudoplatystoma tigrinum}

Ces deux espèces de poissons-chats se distribuent sur tout le bassin amazonien et représentent des nouveaux venus dans le panorama aquacole amazonien en compagnie d'une espèce proche ( $P$. corruscans), mais présente seulement sur le bassin du Paraná et du Sao Francisco. Les tailles maximales de ces espèces se situent autour de 1 à 1,30 m, et la croissance en captivité est compatible avec une production piscicole. Les premiers essais de reproduction sur $P$. fasciatum ont été réalisés il y a plus de 20 ans (Kossowski et Madrid, 1985) et se sont poursuivis depuis lors (Kossowski et Madrid, 1991 ; Padilla Pérez et al., 2001), mais les difficultés rencontrées au cours de l'élevage larvaire n'ont pas permis un développement significatif de l'élevage à l'échelle de la région. Des essais de reproduction ont été réalisés au Brésil sur P. corruscans (Sato et al., 1997), et ils ont permis une production significative et croissante d'alevins. D'après les résultats obtenus, on peut indiquer pour $P$. corruscans un poids d'environ $2 \mathrm{~kg}$ après 12 à 15 mois d'élevage y compris la phase larvaire (Lorena Campos, 2005).

Plus récemment, de nouvelles avancées ont été constatées dans différents pays tant en reproduction qu'en élevage larvaire de $P$. fasciatum (Gervásio Leonardo et al., 2004 ; Nuñez et al., 2008). L'un des principaux problèmes réside dans l'obtention d'alevins entraînés à la consommation d'aliment granulé ou extrudé. Les productions expérimentales réalisées jusqu'ici permettent d'espérer une pro- duction d'alevins d'environ $5 \mathrm{~g}$ de poids moyen après une phase larvaire de 45 jours avec un taux de survie de l'ordre de 30 à $50 \%$. Compte tenu de la relativement grande fécondité de l'espèce (100 000 oufs/kg de femelle), ces taux de survie restent compatibles avec un développement de la production piscicole de ces espèces au cours des prochaines années.

Cependant, il faut déjà relever l'existence d'hybrides interspécifiques au Brésil $(P$. corruscans $\times P$. fasciatum) qui, comme dans le cas du Tambacú, font peser une grave menace sur les populations naturelles, en l'absence de toute étude sur l'état de développement du système reproducteur de ces hybrides. Il est prévisible que le développement de la pisciculture s'accompagne de ces pratiques nouvelles de reproduction et d'élevage larvaire, dont l'avantage par rapport aux souches pures n'a pas été mis en évidence dans la plupart des cas à l'aide de protocoles appropriés, mais repose le plus souvent sur des idées reçues.

\section{Rhamdia quelen}

Le genre Rhamdia est originaire de l'Amazone et de l'Orénoque (Perdices et al., 2002); il est distribué assez largement aujourd'hui sur toute l'Amérique centrale et du Sud et comporterait seulement 11 espèces (Silfvergrip, 1996), alors qu'une centaine avait été décrite antérieurement. Ces espèces atteignent des tailles maximales de l'ordre de 35 à $60 \mathrm{~cm}$ et se rencontrent dans les milieux d'eaux calmes (lacs ou rivières). Elles présentent une activité essentiellement nocturne ou crépusculaire comme beaucoup d'espèces de poissons-chats. Dans le milieu naturel, le régime alimentaire est omnivore (de Carvalho Gomes et al., 2000), ce qui leur confère de bonnes capacités d'adaptation à la consommation d'aliments granulés en captivité. Les premiers essais d'élevage remontent aux années 1980 (Godinho et al., 1978 ; Varela et al., 1982 ; Luchini et Cruz Rangel, 1983). Actuellement, l'espèce la plus utilisée est certainement R. quelen, car ses caractéristiques biologiques (croissance et reproduction notamment) en ont fait un bon candidat non seulement pour la pisciculture en Amazonie, mais également en Amérique centrale et jusqu'en Argentine.

La fécondité apparente se situe entre 50000 et 70000 œufs/kg de femelle, et les œufs sont relativement petits (1,1 à 
1,4 $\mathrm{mm}$ ) avant hydratation. La larve mesure de 4 à $5 \mathrm{~mm}$ à l'éclosion (Varela et al., 1982), ce qui se traduit par un élevage larvaire difficile, les larves pouvant présenter un cannibalisme marqué comme chez Pseudoplatystoma. L'alevinage peut être réalisé en petits étangs (200 à 300 larves $/ \mathrm{m}^{2}$ ), mais il est préférable de travailler en circuit fermé avec un aliment granulé en distribution continue.

Le grossissement peut être fait en étangs, en partant d'alevins d'au moins $5 \mathrm{~cm}$ de longueur. Les meilleurs résultats rapportés oscillent autour de $300 \mathrm{~g}$ (taille commerciale) au bout de 100 jours à des biomasses finales de $0,5 \mathrm{~kg} / \mathrm{m}^{2}$ (Luchini et Quiros, 1990). La croissance est généralement meilleure de 20 à $30 \%$ pour les femelles, les mâles atteignant la maturité sexuelle plus tôt (Araujo-Lima et de Carvalho Gomes, 2005).

Par ailleurs, comme déjà mentionné pour d'autres espèces, il existe au Brésil, des "variétés " qui présenteraient de meilleures performances de croissance et des patrons de couleurs plus appréciés de certains consommateurs locaux, comme par exemple la variété "jundiá-cinza " et "jundiá-albino " obtenus par sélection (Baldisserotto et Radünz Neto, 2005).

Les productions de cette " petite " espèce restent encore faibles (tableau 1), mais son développement devrait se poursuivre, car elle présente de bonnes caractéristiques biotechniques et sa chair est appréciée dans tout le continent sud-américain.

\section{Conclusion}

L'accroissement de la production piscicole au cours des dernières années est sensible même si, pour le moment, la couverture des besoins en poissons d'eau douce est encore largement assurée par la pêche, qui continue de progresser en tonnage annuel, bien que les débarquements de certaines espèces économiquement importantes diminuent (notamment Colossoma, Piractus, Pseudoplatystoma ou Arapaima). L'offre de l'aquaculture est donc venue compenser en partie la raréfaction de ces espèces dans le milieu naturel. Pour continuer à jouer ce rôle et donc à se développer, la pisciculture devra faire face à de nombreuses contraintes : disponibilités en eau et en espace, approvisionnement en matières premières pour la fabrication d'aliments et respect de l'environnement. Dans ce dernier domaine, il convient de souligner que le développement de la pisciculture en Amérique du Sud s'accompagne d'introductions d'espèces étrangères et de pratiques comme l'hybridation interspécifique ou même intergénérique, cette dernière étant en plein développement avec le tambacú. Il est donc probable que cette pratique continuera et même s'amplifiera, malgré les dangers potentiels des échappements de poissons d'élevage dans le milieu naturel et des risques de "pollution génétique " qu'ils constituent pour les espèces et populations sauvages. De façon plus générale, les différentes étapes de domestication-sélection vont conduire à la création de patrimoines génétiques adaptés aux conditions d'élevage (meilleure croissance, meilleure tolérance au stress, etc.), mais aussi de plus en plus divergents de ceux des populations sauvages. Les espèces en élevage ne constitueront donc plus une ressource génétique capable de restaurer, dans leur état initial, des populations naturelles menacées. Cet aspect est à considérer dans le cadre d'opérations de "repeuplement " en utilisant des stocks de géniteurs aussi proches que possible des populations sauvages que l'on souhaite réhabiliter, plutôt que des animaux adaptés aux conditions d'élevage depuis plusieurs générations.

Le développement nécessaire et souhaitable de la pisciculture devra donc s'accompagner de règles de conduite claires et applicables afin de permettre une pisciculture raisonnée et durable, respectueuse du milieu tant du point de vue des ressources hydriques que biologiques. Cela est éminemment plus important dans une zone où la biodiversité est encore extrêmement riche et où tout " accident " pourrait mettre en péril de nombreuses espèces sauvages. Pour relever ce défi, il faudra concilier l'utilisation d'espèces locales de mieux en mieux domestiquées et donc de plus en plus éloignées génétiquement des populations sauvages tout en sauvegardant autant que possible l'indispensable réservoir des ressources génétiques naturelles dont certaines sont déjà gravement menacées.

\section{Références}

Araujo-Lima CARM, de Carvalho Gomes L. Tambaqui (Colossoma macropomum). In : Baldisserotto B, Gomes L, eds. Especies nativas para piscicultura no Brasil. Santa Maria (Brasil) : UFSM, 2005.
Baldisserotto B, Radünz Neto J. Jundiá (Rhamdia sp.). In: Baldisserotto B, Gomes $L$, eds. Especies nativas para piscicultura no Brasil. Santa Maria (Brasil) : UFSM, 2005.

Baras E, Ndao M, Maxi MYJ, et al. Sibling cannibalism in dorada under experimental conditions. I. Ontogeny, dynamics, bioenergetics of cannibalism and prey size selectivity. J Fish Biol $2000 ; 57: 1001-20$.

Calcagnotto D, de Almeida-Toledo L, Bernardino G, de Almeida Toledo-Filho S. Biochemical genetic characterization of $F 1$ reciprocal hybrids between neotropical pacu (Piaractus mesopotamicus) and tambaqui (Colossoma macropomum) reared in Brazil. Aquaculture $1999 ; 174: 51-7$.

Chu-Koo F, Dugué R, Alvan Aguilar M, et al. Gender determination in the Pirarucu or Paiche (Arapaima gigas) using plasma vitellogenin, 17ß-estradiol, and 11-ketotestosterone levels. Fish Physiol Biochem 2008: http://dx. doi.org/10.1007/s10695-008-9211-8.

de Borba MR, Machado Fracalossi D, Edivaldo Pezzato L, Menoyo D, Bautista J. M. Growth, lipogenesis and body composition of piracanjuba (Brycon orbignyanus) fingerlings fed different dietary protein and lipid concentrations. Aquat Living Resour 2003 ; 16 : 362-9.

de Carvalho Gomes L, Ineu Golombieski J, Chippari Gomes AR, Baldisserotto B. Biologia do jundiá Rhamdia quelen (Teleostei, Pimelodidae). Ciência Rural, Santa Maria 2000; 30 : 179-85.

FAO. Inland fisheries management in Latin America and its contribution to food security and poverty alleviation. FAO/COPESCAL/X/05/ 3E. Rome : FAO, 2005.

Feiden A, Hayashi C. Desenvolvimento de juvenis de Piracanjuba (Brycon orbignyannus), Vallencienes (1849) (Teleostei: characidae) em tanques experimentais fertilizados com adubação orgânica. Ciênc Agr 2005 ; 26 : 591-600.

Freato TA, Fonseca de Freitas RT, dos Santos VB, Rosa Logato PV, de Mendonça Viveiros A. Efeito do peso de abate nos rendimentos do processamento da piracanjuba, Brycon orbignyanus (Valenciennes, 1849). Ciênc Agr $2005 ; 29$ : 676-82.

Ganeco LN, Nakaghi LSO, Urbinati EC, Dumont Neto R, Vasques LH. Análise morfológica do desenvolvimento ovocitário de piracanjuba, Brycon orbignyanus, durante o ciclo reprodu tivo. Bol Inst Pesca Sao Paulo 2001; 27 : 131-8.

Gervásio Leonardo AF, Romagosa E, Borella MI, Batlouni SR. Induced spawning of hatchery-raised Brazilian catfish, cachara Pseudoplatystoma fasciatum (Linnaeus, 1766). Aquaculture 2004 ; 240 : 451-61.

Godinho HM, Basile-Martins MA, Fenerich N Narahara MY. Desenvolvimento embrionário e larval de Rhamdia hilarii (Valenciennes, 1840) (Siluriformes, Pimelodidae). Rev Bras Biol São Carlos 1978; 38 : 151-6.

Henn AW. The range of size in the vertebrates. Am Nat $1912 ; 46$ : 157-62.

Junk WJ, Soares MGM, Bayley PB. Freshwater fishes of the Amazon River basin : their biodiversity, fisheries, and habitats. Aquat Ecosyst Health Manage 2007; 10 : 153-73.

Kossowski C, Madrid F. Ensayo de la reproducción inducida en el bagre rayado cabezón Pseudoplatystoma fasciatum (Linnaeus, 1766) (Pisces, Siluriformes). Acta Cient Venez 1985 ; $36: 284-5$ 
Kossowski C, Madrid F. Observaciones de los estadios embrionario y larval del bagre rayado cabezón Pseudoplatystoma fasciatum (Linnaeus, 1766) (Pisces, Siluriforms). BioLlania $1991 ; 8: 9-15$

Lorena Campos J. O cultivo do pintado, Pseudoplatystoma coruscans (Spix é Agassiz, 1829). In : Baldisserotto B, Gomes L, eds. Especies nativas para piscicultura no Brasil. Santa Maria (Brasil) : UFSM, 2005.

Luchini L, Cruz Rangel C. Uso de gonadotrofina coriónica humana en la reproducción artificial de Rhamdia sapo. Rev Asoc Cienc Nat Litor St Tome 1983; 14 : 87-92.

Luchini LM, Quiros R. Cage culture of south american catfish (Rhamdia sapo), preliminary results in the Salto Grande reservoir (Argentina). J Aquac Trop $1990 ; 5: 163-72$.

Lundberg JG, Kottelat M, Smith GR, Stiassny MLJ, Gill AC. So many fishes, so little time: an overview of recent ichthyological discovery in continental waters. Ann Missouri Bot Garden 2000 ; 87 : 26-62.

Mateus L, Estupiñán GMB. Fish stock assessment of piraputanga Brycon microlepis in the Cuiabá River basin, Pantanal of Mato Grosso, Brazil. Braz J Biol 2002 ; 62 : 165-70.

Mazzotti Santamaria F, Araujo Antunes S. Coloração e rendimento do filé de piracanjuba, Brycon orbignyanus (Valenciennes, 1849), (Pisces, Characidae) silvestre e criada em cativeiro. Bol Inst Pesca, São Paulo 1999; 25 : 27-30.
Nuñez J, Dugué R, Corcuy Arana N, et al. Induced breeding and larval rearing of Surubí, Pseudoplatystoma fasciatum (Linnaeus, 1766), from the Bolivian Amazon. Aquac Res 2008; $39:$ 764-76.

Padilla Pérez PP, Alcántara Bocanegra $F$, Ismiño Orbe R. Reproducción inducida de la doncella Pseudoplatystoma fasciatum y desarrollo embrionario-larval. Folia Amazonica $2001 ; 12: 141-54$.

Perdices A, Bermingham E, Montilla A, Doadrio I. Evolutionary history of the genus Rham dia (Teleostei : Pimelodidae) in Central America. Mol Phyl Evol 2002 ; 25 : 172-89.

Pereira-Filho M, Gandra AL, Bordinhon AM, et al. Arapaima gigas: Notas sobre seu Cultivo no INPA. In : Alcántara Bocanegra F, Montreuil Frias V, eds. Seminario taller internacional de manejo de paiche o pirarucu. Iquitos (Perú) : IIAP, 2003.

Petrere $M$, Barthem RB, Córdoba EA, Gómez $B C$. Review of the large catfish fisheries in the upper Amazon and the stock depletion of piraíba (Brachyplatystoma filamentosum Lichtenstein). Rev Fish Biol Fisher 2004; 14: 403-14.

Rebaza Alfaro M, Rebaza Alfaro C, Deza Taboada S. Observaciones de la reproduction de paiche Arapaima gigas (Cuvier) en ambientes controlados en el IIAP Ucayali. In: Alcántara Bocanegra F, Montreuil Frias V, eds. Seminario taller internacional de manejo de paiche o pirarucu. Iquitos (Perou) : IIAP, 2003.
Saavedra Rojas ÉA, Quintero Pinto LG, Landines Parra MÁ. Aspectos Reproductivos. In : Sanabria AI, Beltran IC, Daza PV, eds. Biología y cultivo del pirarucú Arapaima. Bogotá (Colombia) : INCODER/UNC, 2005.

Sato Y, Cardoso EL, Sallum WB, Godinho HP Induçao experimental da desove do surubim Pseudoplatystoma coruscans. In: Miranda MOT, ed. Surubim. Belo Horizonte (Brasil): IBAMÁ, 1997.

Silfvergrip AMC. A systematic revision of the neotropical catfish genus Rhamdia (Teleostei, Pimelodidae). PhD Thesis. Department of Zoology, Stockholm University and Department of Vertebrate Zoology, Swedish Museum of Natural History, Stockholm (Sweden), 1996.

Tyler CD. The River Napo. Geogr J 1894 ; 3 : 476-84.

Varela Z, Fischer K, Fabiano G. Reproducción artificial del bagre negro Rhamdia sapo. Proyecto FAO/PNUD/URU/78/005. Montevideo (Uruguay) : sn, 1982.

Welcomme RL. River fisheries. FAO Fisheries Technical Paper. Rome: Food and Agriculture Organization, 1985.

Woynarovich E, Horváth L. La reproduction artificielle des poissons en eau chaude: manuel de vulgarisation. FAO Doc Tech Pêches. Rome: Food and Agriculture Organization, 1981. 